\title{
MODELAGEM DE VARIÁVEIS MORFOLÓGICAS EM MUDAS DE Eucalyptus dunnii
}

\author{
Marcos Felipe Nicoletti ${ }^{1}$, Marcio Carlos Navroski ${ }^{1}$, Nilton Sergio Novack Junior ${ }^{2}$ \\ ${ }^{1}$ Universidade do Estado de Santa Catarina, Departamento de Engenharia Florestal, Lages, Santa Catarina, Brasil - \\ marcos.nicoletti@udesc.br; marcio.navroski@udesc.br \\ ${ }^{2}$ Universidade do Estado de Santa Catarina, Curso de Engenharia Florestal, Lages, Santa Catarina, Brasil - nsnovack.efl@gmail.com
}

Recebido para publicação: 12/08/2014 - Aceito para publicação: 22/05/2015

\begin{abstract}
As variáveis morfológicas de mudas florestais podem ser indicativos de sua qualidade, sendo de grande importância para o sucesso de empreendimentos florestais. Nesse contexto, o objetivo do estudo foi correlacionar as variáveis operacionalmente mensuráveis (diâmetro do colo e altura) com as de mensuração destrutiva (área foliar e comprimento de raiz) de mudas de E. dunnii, de modo a formular equações que forneçam estimativas precisas dessas variáveis. Para isso, avaliou-se 77 mudas com 100 dias de idade. As equações foram formuladas através da metodologia "stepwise" para seleção da melhor combinação entre 45 transformações matemáticas das variáveis operacionalmente mensuráveis. As equações foram avaliadas por estatísticas de ajuste e precisão, além de posterior validação por meio de um teste de aderência, a um nível de confiança de $99 \%$, utilizando 50 mudas independentes. Observou-se alta correlação entre as variáveis. Para área foliar, obteve-se coeficiente de determinação ajustado de 0,91 e erro padrão da estimativa de 15,2\%. Para comprimento de raiz, coeficiente de determinação ajustado de 0,76 e erro padrão da estimativa de $22,5 \%$. Os dois casos não apresentaram tendenciosidade residual e foram validadas. Diante do exposto, é possível formular equações para obter estimativas de área foliar e comprimento de raiz em $E$. dunnii.

Palavras-chave: Qualidade de mudas; área foliar; comprimento de raiz; modelos matemáticos.
\end{abstract}

Resumo

\begin{abstract}
Modeling of morphological variables in Eucalyptus dunnii seedlings. The morphological variables of forest seedlings may be indicative of its quality, having great importance to the success of forest enterprises. In this context, the aim of this study was to correlate the operationally measurable variables (stem diameter and height) with a destructive measurement (leaf area and root length) of $E$. dunnii seedlings in order to formulate equations that provide accurate estimates of these variables. For this, 77 seedlings were evaluated at 100 days old. The equations were formulated using the methodology "stepwise" for selecting the best combination of mathematical transformations of 45 operationally measurable variables. These equations were evaluated by statistical adjustment and precision, and subsequent validation by means of adherence test, with a confidence level of $99 \%$, using 50 independent seedlings. The results indicated high correlation between the variables. For leaf area, the obtained adjusted coefficient of determination was 0.91 and standard error of estimate $15.2 \%$. To root length, the adjusted coefficient of determination was 0.76 and standard error estimate of $22.5 \%$. The two cases presented no residual bias and had been validated. as result, it is possible to formulate equations to obtain estimates of leaf area and root length in E. dunnii.

Keywords: Seedlings quality; leaf area; root length; mathematical models.
\end{abstract}

\section{INTRODUÇÃO}

A alta produtividade dos reflorestamentos e florestamentos comerciais depende da qualidade das mudas que vão para campo. Qualidade esta que é dependente de vários fatores que geram influência no crescimento e aclimatação das mudas. Basicamente, estes fatores refletem diretamente nos custos de implantação de um povoamento, pois quanto mais rápido o crescimento inicial, menor pode ser a necessidade do controle de plantas daninhas, bem como a aclimatação que pode reduzir a taxa de replantio. Em suma, a sobrevivência, o estabelecimento, a frequência dos tratos culturais e o crescimento inicial das florestas são características dependentes da qualidade de mudas utilizadas (BONFIM, 2007). Portanto, é necessário a avaliação de todas as variáveis que podem gerar influência na qualidade das mudas. 
Existem diversas variáveis que geram influência na qualidade das mudas. As variáveis mais utilizadas na determinação do padrão de qualidade de mudas de espécies arbóreas são a altura da planta, diâmetro do colo, massa seca total, massa seca da parte aérea, massa seca de raízes e algumas razões entre essas variáveis (AGUIAR et al., 2011). Segundo Carneiro (1995), as variáveis que determinam a qualidade das mudas podem ser também, de natureza fenotípica (variáveis morfológicas) e de natureza interna (variáveis fisiológicos), tais como potencial hídrico, estado nutricional e ecofisiologia de raízes. Porém, as variáveis morfológicas não devem ser utilizados de maneira isolada para determinar a qualidade de mudas devido à forte relação entre as variáveis, pois quando tiver um desequilíbrio entre eles pode gerar inferioridade na qualidade, por exemplo, mudas muito altas com diâmetros muito inferiores podem caracterizar mudas com grandes chances de sofrer tombamento (LIMA et al.,2006).

É necessário entender como cada variável se comporta e gera influência na qualidade das mudas para poder interferir e aumentar o rendimento do empreendimento florestal. As variáveis morfológicas como altura e diâmetro do colo são facilmente mensuráveis e podem dar respostas sobre o vigor e funcionalidade das mudas (ARAÚJO, 2009), entretanto, variáveis destrutivas como massa seca aérea e radicular e área foliar são importantes para expressar qualidade da muda, porém são de difícil mensuração.

A altura das mudas mede a capacidade de fotossíntese e a área de transpiração, tem relação com a qualidade de mudas, porém, mudas maiores em altura podem não representar uma boa qualidade (DURVEA, 1985), diante do exemplo dado anteriormente. O diâmetro do colo é a característica mais observada para indicar a capacidade de sobrevivência das mudas no campo (D'AVILA et al., 2011). Existem também outras variáveis que quando correlacionadas com as duas variáveis supracitadas fornecem respostas mais precisas. O diâmetro do colo tem correlação com o tamanho médio do sistema radicular, bem como a altura tem relação com o número de folhas e a área foliar (ELOY et al., 2013).

A percepção da relação entre as variáveis facilmente mensuráveis de forma não destrutiva, como diâmetro do colo e altura de mudas, com as variáveis de difícil obtenção ou que causam a destruição das mudas é de grande valia. Medições diretas de comprimentos de raízes são, operacionalmente, cansativas e consomem muito tempo (CARNEIRO, 1995). Assim, é possível ajustar modelos que forneçam estimativas seguras das variáveis de maior dificuldade de medição. Logo, obter dados estimados confiáveis de forma não destrutiva por meio da modelagem matemática é uma ferramenta importante para aperfeiçoar a produção florestal. Desde que a sua aplicação seja em mudas de mesma espécie, idade e em características climáticas semelhantes às mudas utilizadas para formular as equações.

Portanto, é visível a necessidade da criação de modelos de regressão que correlacionem as variáveis, com base nisso é possível estimar as variáveis de difícil obtenção, que são indicativos precisos de qualidade das mudas de forma mais usual. O objetivo do estudo foi correlacionar as variáveis operacionalmente mensuráveis (diâmetro do colo e altura) com as de mensuração destrutiva (área foliar e comprimento de raiz) de mudas de $E$. dunnii, de modo a formular equações que forneçam estimativas precisas dessas variáveis.

\section{MATERIAL E MÉTODOS}

O experimento foi conduzido em casa de vegetação, no viveiro florestal do Centro de Ciências Agroveterinárias (CAV), da Universidade do Estado de Santa Catarina, localizado no município de Lages, Santa Catarina. O clima da região é caracterizado temperado subtropical com invernos e verões amenos $(\mathrm{Cfb})$. A precipitação média anual é de $1500 \mathrm{~mm}$ com temperatura média anual de $14,3{ }^{\circ} \mathrm{C}$, sendo a máxima registrada de $36{ }^{\circ} \mathrm{C}$ e mínima registrada de $-7^{\circ} \mathrm{C}$, com altitude média de $905 \mathrm{~m}$.

As sementes de Eucalyptus dunnii utilizadas são originárias de Área de Produção de Sementes da empresa Bentec (Rio do Sul, SC), da qual foi feita a aquisição do material. As sementes foram acondicionadas em embalagem de plástico semipermeável (90 micras de espessura) e armazenadas em câmara fria $\left(\mathrm{T}=8^{\circ} \mathrm{C}\right.$; UR $\left.=85 \%\right)$ por 12 meses. A semeadura foi realizada diretamente nos tubetes de $110 \mathrm{~cm}^{3}$, utilizando o substrato comercial Tecnomax ${ }^{\circledR}$ para plantas. Segundo o fabricante, o substrato é composto por turfa, vermiculita expandida, casca de Pinus sp. e carvão vegetal. Apresentando as seguintes características: $\mathrm{pH}=6,0( \pm 0,5)$; condutividade elétrica $=0,7( \pm 0,3) \mathrm{mS} \mathrm{cm}^{-1}$; densidade $=$ $500 \mathrm{~kg} \mathrm{~m}^{-3}$; capacidade de retenção de água - CRA $(\mathrm{p} / \mathrm{p})=150 \%$ e umidade máxima $(\mathrm{p} / \mathrm{p})=50 \%$. O substrato foi homogeneizado com as devidas doses de Osmocote ${ }^{\circledR}$ e em seguida os tubetes foram distribuídos sobre grades metálicas, utilizando $100 \%$ do espaço nos primeiros 60 dias e passando a ocupar $50 \%$ do espaço dos 60 aos 100 dias. As plantas foram irrigadas diariamente duas vezes ao dia, totalizando uma lâmina de irrigação de $8 \mathrm{~mm} \mathrm{dia}^{-1}$. Foram coletados dados de 77 mudas com 100 dias idade. 


\section{Coleta e processamento dos dados}

As variáveis coletadas para análise foram: altura total (h), diâmetro do colo (dc), área foliar (af) e comprimento de raiz (cr). A altura foi mensurada com régua graduada em centímetros e o diâmetro do colo com auxílio de um paquímetro eletrônico.

Para determinar a área foliar e o comprimento de raízes foram retiradas folhas e raízes, respectivamente, e distribuídas manualmente sobre uma folha de papel branco no tamanho A4. Com o auxílio de uma câmera digital, apoiada em um suporte de altura fixa $(0,5 \mathrm{~m})$, obtiveram-se imagens digitais. Utilizando o programa UTHSCSA, Image Tool for Windows version 3.00® (2002), com a determinação da escala, todas as imagens foram calibradas para posterior mensuração. Este programa detecta as folhas e fornece a área $\left(\mathrm{cm}^{2}\right)$ das mesmas e o comprimento $(\mathrm{cm})$ das raízes.

Foi verificado se os dados seguem a distribuição normal por meio do teste de normalidade de Kolmogorov-Smirnov. A tabulação e o processamento dos dados foram realizados no software Microsoft Excel.

\section{Modelagem}

Após a coleta dos dados e verificação do comportamento normal, foi aplicado o teste de correlação de Pearson com a finalidade de conhecer as variáveis que mais se correlacionam.

Com a finalidade de se obter uma equação para determinação de área foliar, e outra para comprimento de raiz, foram avaliadas diversas transformações matemáticas (Tabela 1) para verificar qual ou quais tiveram uma melhor significância para tais estimativas.

Tabela 1. Transformações matemáticas das variáveis independentes.

Table 1. Mathematical transformations of the independent variables.

\begin{tabular}{ccccc}
\hline \multicolumn{5}{c}{ Transformações matemáticas } \\
\hline $\mathbf{h}$ & $\mathrm{h}^{2}$ & $\mathrm{~h}^{3}$ & $\sqrt{\mathrm{h}}$ & $\frac{1}{\sqrt{\mathrm{h}}}$ \\
$\frac{\mathbf{h}}{\mathbf{h}^{2}}$ & $\frac{1}{\mathrm{~h}^{3}}$ & $\ln \mathrm{h}$ & $\ln \mathrm{h}^{2}$ & $\frac{1}{\ln \mathrm{h}}$ \\
$\mathbf{h} \cdot \mathbf{d c}$ & $\mathrm{h}^{2} \cdot \mathrm{dc}$ & $\mathrm{h}^{3} \cdot \mathrm{dc}$ & $\frac{1}{\mathrm{~h}} \cdot \mathrm{dc}$ & $\frac{1}{\mathrm{~h}^{2}} \cdot \mathrm{dc}$ \\
$\mathbf{l n} \mathbf{h} \cdot \mathbf{d c}$ & $\frac{1}{\mathrm{dc} \cdot \mathrm{h}}$ & $\frac{1}{\mathrm{dc} \cdot \mathrm{h}^{2}}$ & $\frac{1}{\mathrm{dc} \cdot \mathrm{h}^{3}}$ & $\frac{1}{\mathrm{~h}} \cdot \frac{1}{\mathrm{dc}}$ \\
$\frac{\mathbf{1}}{\mathbf{h}^{3}} \cdot \frac{\mathbf{1}}{\mathbf{d c}}$ & $\frac{1}{\mathrm{dc}}$ & $\mathrm{dc}$ & $\mathrm{dc}^{2}$ & $\mathrm{dc}$ \\
$\mathbf{l n} \mathbf{d c} \mathbf{c}^{2}$ & $\frac{1}{\ln \mathrm{dc}}$ & $\sqrt{\mathrm{dc}}$ & $\frac{1}{\sqrt{\mathrm{dc}}}$ & $\ln (\mathrm{h}+\mathrm{dc})$ \\
$\mathbf{d c}+\mathbf{h}^{2}$ & $\frac{\mathrm{dc}}{\mathrm{h}}$ & $\mathrm{dc} \cdot \mathrm{h}$ & $\frac{1}{\frac{1}{\mathrm{dc}^{2}+\mathrm{h}^{2}}}$ & $\mathrm{dc}$ \\
$\frac{\mathbf{1}}{\mathbf{h}}$ & $\frac{1}{\sqrt{\mathrm{h}^{2}+\mathrm{dc}^{2}}}$ & $\frac{1}{\mathrm{~h}^{3}} \cdot \mathrm{dc}$ & $\frac{1}{\mathrm{~h}^{2}} \cdot \frac{1}{\mathrm{dc}}$ & $\ln \mathrm{dc}$ \\
\hline
\end{tabular}

$d c$ : Diâmetro do colo; $h$ : Altura total da muda; $\ln$ : logarítimo neperiano.

Foram selecionadas as transformações matemáticas que conferiram maior significância às equações pelo método de seleção de variáveis stepwise. Tal método define qual a combinação de transformações matemáticas forma a equação de maior ajuste e precisão, com base na significância de cada uma. A análise foi realizada no software Statgraphics Centurion ${ }^{\circledR}$. As equações propostas foram avaliadas quanto ao ajuste com base no coeficiente de determinação ajustado, e quanto à precisão com base na dispersão gráfica dos resíduos e também através do erro padrão da estimativa na sua forma relativa.

As equações passaram por teste de validação para que houvesse garantia de que os valores estimados não apresentassem diferença estatística significativa quando comparado com os valores observados. Para tanto, aplicou-se o teste não-paramétrico Qui-Quadrado $\left(\chi^{2}\right)$ a um nível de confiança de $99 \%$. Para aplicar o teste, utilizou-se dados de 50 mudas de E. dunnii, que apresentaram as características de validação propostas por

FLORESTA, Curitiba, PR, v. 45, n. 4, p. 809 - 818, out. / dez. 2015.

Nicoletti, M. F. et al.

ISSN eletrônico 1982-4688 / ISSN impresso 0015-3826

DOI: $10.5380 /$ rf.v45i4.37454 
Prodan et al. (1997), os quais sugerem que a amostra para validação deve ser independente dos dados utilizados no ajuste da equação, e devem cobrir toda a amplitude das variáveis observadas.

\section{RESULTADOS E DISCUSSÃO}

Todas as variáveis analisadas apresentaram comportamento normal conforme o teste de Kolmogorov-Smirnov, o que sustenta a validade de todas as análises subsequentes. A dispersão dos pontos residuais ao longo da linha de normalidade confirmam graficamente o resultado (Figura 1).
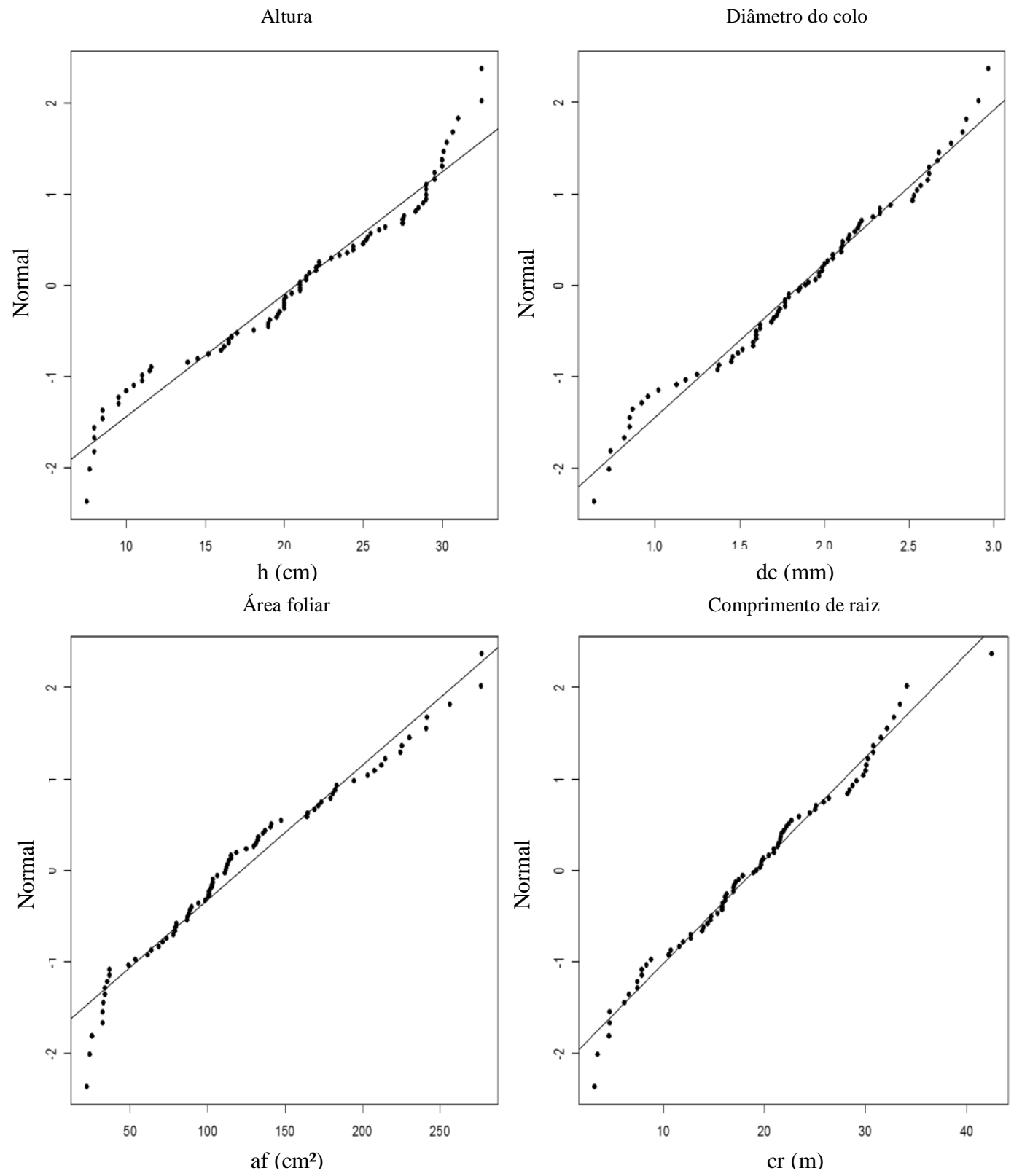

Figura 1. Dispersão residual de normalidade das variáveis analisadas segundo teste de KolmogorovSmirnov.

Figure 1. Normality residual dispersion of the analyzed variables using the Kolmogorov-Smirnov test. 
É visível a tendência dos pontos seguirem a linha normal em todas as variáveis. Existe uma porção ínfima nos extremos superiores em todas as variáveis que destoaram levemente da linha normal, porém, tais porções não desclassificam a normalidade dos dados.

A confiabilidade das estimativas realizadas pelas equações se dá no intervalo correspondente ao mínimo e máximo das respectivas variáveis independentes de cada uma das equações propostas. Tais informações, além de algumas estatísticas descritivas dos dados em análise estão dispostas na tabela 2.

Verifica-se que as mudas selecionadas para a análise cobrem uma variação ampla de todas as etapas de produção para o diâmetro do colo e a altura. Na prática, isso retrata a flexibilidade do uso das equações para mudas que se enquadrem em valores entre o máximo e o mínimo apresentados na tabela anterior. Portanto, é possível estimar as variáveis de difícil obtenção de mudas em dimensões dentro de suas características ideais de introdução à campo, geralmente com diâmetro em torno de $2 \mathrm{~mm}$ e altura por volta de $30 \mathrm{~cm}$, segundo os critérios de qualidade de mudas de Wendling e Dutra (2010).

Tabela 2. Estatística descritiva das variáveis mensuradas em mudas de Eucalyptus dunnii aos 100 dias após semeadura.

Table 2. Descriptive statistics of variables measured in Eucalyptus dunnii at 100 days after sowing.

\begin{tabular}{lccccc}
\hline Variável & Média & Desvio Padrão & Mínimo & Máximo & CV \\
\hline Altura $(\mathrm{cm})$ & 20,7 & 7,2 & 7,5 & 32,5 & $34,5 \%$ \\
Diâmetro do colo $(\mathrm{mm})$ & 1,8 & 0,6 & 0,6 & 3,0 & $31,6 \%$ \\
Área Foliar $\left(\mathrm{cm}^{2}\right)$ & 119,3 & 66,5 & 13,8 & 276,7 & $55,7 \%$ \\
Comprimento de raiz $(\mathrm{m})$ & 18,9 & 8,6 & 3,2 & 42,5 & $45,8 \%$ \\
\hline
\end{tabular}

CV: Coeficiente de variação.

Conforme matriz de correlação de Pearson (Tabela 3), observa-se que a variável dependente área foliar tem maior correlação com o diâmetro do colo, o mesmo se repete para a variável dependente comprimento de raiz. Porém, a variável altura também possui uma correlação considerável com as variáveis dependentes.

Tabela 3. Matriz de correlação entre as variáveis morfológicas analisadas em mudas de Eucalyptus dunnii aos 100 dias após semeadura.

Table 3. Correlation matrix of the morphologic variables analyzed in Eucalyptus dunnii at 100 days after sowing.

\begin{tabular}{lcccc}
\hline Variáveis & Altura & Diâmetro do colo & Área foliar & Comprimento de raiz \\
\hline Altura & 1 & - & - & - \\
Diâmetro do colo & 0,9107 & 1 & - & - \\
Área foliar & 0,8763 & 0,9006 & 1 & - \\
Comprimento de raiz & 0,7782 & 0,8449 & 0,8394 & 1 \\
\hline
\end{tabular}

A correlação existente entre as variáveis proporciona viabilidade de formulação de equações que tenham a finalidade de estimar variáveis que não são passíveis de mensuração não destrutiva, uma vez que as variações de área foliar e comprimento de raiz podem ser explicadas pelo diâmetro do colo e altura. Conforme o teste de correlação, pode-se destacar o diâmetro do colo como peça importante na predição das variáveis de difícil mensuração.

O diâmetro do colo é amplamente aceito como uma variável confiável, uma vez que oferece maior correlação com a sobrevivência de mudas a campo, e de fácil mensuração para determinar a qualidade de mudas (CARNEIRO, 1995). Resultado semelhante a este estudo foi verificado por Rudek et al. (2013) em Eucalyptus grandis. Conforme os autores foi possível presumir que a determinação da área foliar associada ao diâmetro do coleto seria uma variável consistente para a determinação da qualidade de mudas de $E$. grandis apresentando correlação de 0,78 entre essas duas.

Apesar da altura também apresentar uma forte correlação com as demais variáveis, principalmente diâmetro do colo e área foliar, se for utilizada de forma isolada pode fornecer um padrão de qualidade errôneo. Isso porque pode ser muito influenciada por variações ambientais, densidade populacional e pelos níveis nutricionais da muda, os quais variam de acordo com a fertilização fornecida. Por exemplo, o aumento nos teores de nitrogênio pela adubação nitrogenada pode estimular o

FLORESTA, Curitiba, PR, v. 45, n. 4, p. 809 - 818, out. / dez. 2015.

Nicoletti, M. F. et al.

ISSN eletrônico 1982-4688 / ISSN impresso 0015-3826

DOI: 10.5380/rf.v45i4.37454 
estiolamento da muda (CARNEIRO, 1995). O crescimento estiolado implicaria em mudas cuja altura padrão seria atingida precocemente, o que implicaria no envio de mudas de baixa qualidade para o campo. Ainda, pode-se evitar o alongamento excessivo do caule de mudas impróprias para o comércio ou plantio no campo (mudas velhas) com a aplicação de cloreto de potássio $(\mathrm{KCl})$, resultando em mudas fisiologicamente mais velhas, mas com altura de mudas jovens.

\section{Equações para área foliar e comprimento de raiz}

Observando as correlações existentes entre as variáveis e aplicando os critérios e as metodologias supracitadas, foi possível obter equações para estimativa das variáveis em questão.

A área foliar é uma variável de difícil mensuração e de conhecimento extremamente importante (RUDEK et al., 2013), pois é responsável pela grande área fotossinteticamente ativa na muda, resultando no crescimento em altura e diâmetro do colo. O comprimento radicular também é uma variável importante, pois quanto maior o desenvolvimento radicular, principalmente raízes finas, maior será a absorção de água e nutrientes, resultando no crescimento da planta. Para a determinação da estimativa de área foliar e comprimento radicular por meio das variáveis altura e diâmetro do colo o procedimento de stepwise resultou a equação 1 e 2 , respectivamente (Tabela 4).

As estatísticas de ajuste e precisão da equação expressas na (Tabela 4 e Figura 2) demonstram que entre todas as combinações propostas, a transformação $h \cdot d c$ foi a que melhor explicou a variação apresentada pela área foliar.

Tabela 4. Estatística de ajuste e precisão das equações.

Table 4. Statistical adjustment and accuracy of equations.

\begin{tabular}{cccc}
\hline & Equações & $\mathbf{R}_{\mathbf{a j}}$ & $\mathbf{S}_{\mathbf{y x}}(\boldsymbol{\%})$ \\
\hline 1 & $a f=11,9334+2,5261 \cdot(h \cdot d c)+\varepsilon_{i}$ & 0,9162 & 15,2 \\
2 & $c r=-2,0471-0,00028 \cdot h^{3}+4,3045 \cdot \ln (h) \cdot d c+\varepsilon_{i}$ & 0,7606 & 22,5 \\
\hline
\end{tabular}

$\mathrm{R}^{2}{ }_{\mathrm{aj}}$ : Coeficiente de determinação ajustado; $\mathrm{S}_{\mathrm{yx}}$ : Erro padrão da estimativa relativo; $a f$ : área foliar $\left(\mathrm{cm}^{2}\right) ; c r$ : comprimento de raiz (m); $h$ : altura total da muda $(\mathrm{cm}) ; d c$ : diâmetro do colo $(\mathrm{mm}) ; \varepsilon_{i}$ : erro de estimativa.

Deve-se observar que as variáveis dependentes da forma em que foram inseridas na equação apresentaram um ajuste considerável, uma vez que quanto menor for a diferença entre a soma dos quadrados da regressão em relação à soma dos quadrados total (DRAPER; SMITH, 1998), considera-se que as estimativas propostas pela equação possuem uma aderência aceitável com relação aos valores observados. Tal comportamento é representado pelo coeficiente de determinação ajustado, que para as estimativas da variável em questão, pode-se considerar que a proporção da variação apresentada pela área foliar pode ser explicada em $91,6 \%$ pela equação proposta.

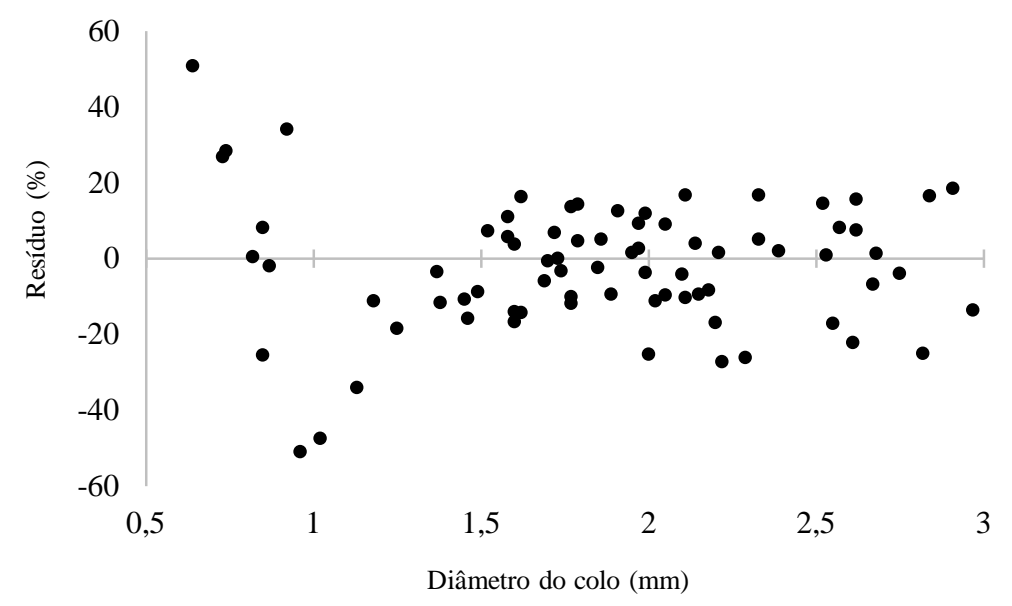

Figura 2. Distribuição residual das estimativas da equação de área foliar para mudas de E. dunnii. Figure 2. Distribution of residual estimates of leaf area equation for seedlings of $E$. dunnii. 
Para atestar a confiabilidade das estimativas, pode-se observar que o erro padrão da estimativa está em um percentual aceitável, uma vez que a área foliar é uma variável de difícil mensuração e de grande importância.

As estimativas da equação tiveram tendência à um percentual residual mais elevado no terço inferior do eixo das abcissas, ou seja, existem maiores superestimativas ou subestimativas de área foliar em mudas de $0,5 \mathrm{~mm}$ a aproximadamente $1,5 \mathrm{~mm}$ de diâmetro do colo. Tais resíduos não geram redução da confiabilidade dos dados, uma vez que os pontos mais discrepantes estão no máximo em torno de $\pm 50 \%$, além de que são poucos pontos que estão acima de $\pm 20 \%$ do erro. Os menores resíduos estão dispersos entre $1,5 \mathrm{~mm}$ e $2,5 \mathrm{~mm}$ de diâmetro, esse comportamento revela maior atratividade prática da equação, já que o diâmetro do colo ideal para implantação das mudas de $E$. dunnii a campo é de aproximadamente $2 \mathrm{~mm}$ (WENDLING; DUTRA, 2010). Utilizar estimativas precisas de área foliar é um ponto chave para assegurar a qualidade das mudas que vão ser implantadas.

Já para comprimento de raiz, observa-se que as transformações matemáticas que mais se adequaram à questão proposta foram o cubo da altura $\left(h^{3}\right)$ e o logaritmo neperiano da altura multiplicado pelo diâmetro do colo $(\ln (h) \cdot d c)$. A confiabilidade da equação está expressa nas estatísticas de ajuste e precisão descritas na tabela 4 e figura 2.

Com base no coeficiente de determinação, constata-se um comportamento semelhante à equação de área foliar, porém um pouco menor. Fato que é explicado pela própria correlação entre as variáveis ser inferior com relação à correlação existente entre área foliar e as variáveis dependentes. Porém, trata-se de um coeficiente de determinação aceitável, uma vez que mais de $76 \%$ da variação apresentada pelo comprimento de raiz está sendo explicada pelas variáveis dependentes.

A porcentagem de erro padrão da estimativa apresentada também pode ser considerada aceitável, uma vez que a coleta de dados é onerosa. Uma equação que estime com essa precisão fornece dados que podem ser utilizados com confiabilidade. A dispersão dos resíduos está representada no gráfico presente na figura 2.

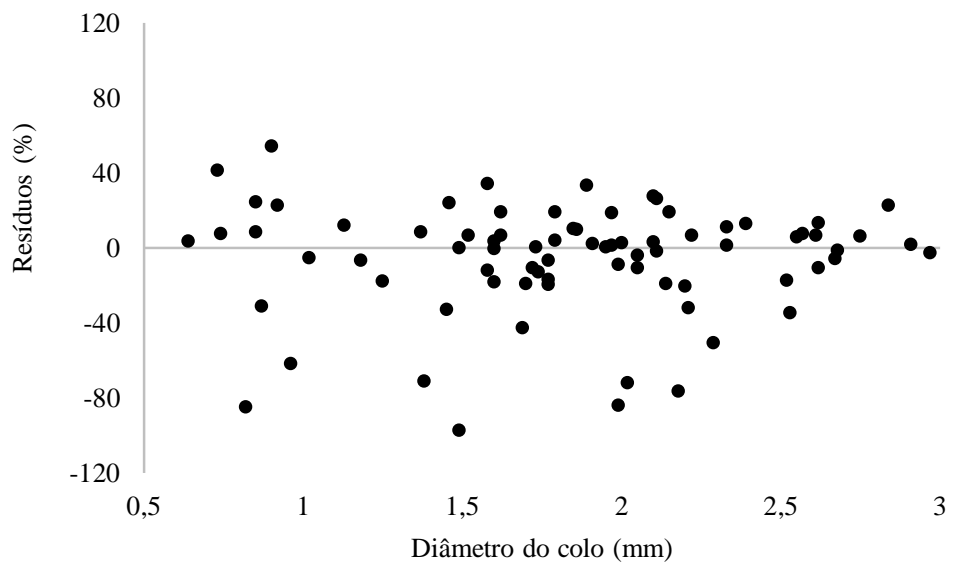

Figura 2. Distribuição residual das estimativas da equação de comprimento de raiz para mudas de $E$. dunnii.

Figure 2. Distribution of residual estimates of root length equation for seedlings of E. dunnii.

A amplitude residual apresentada pela equação, apesar de maior, a torna válida, uma vez que a obtenção desses dados só se dá de maneira destrutiva. A dispersão dos resíduos demonstram que a equação tem maior tendência a superestimar os valores de comprimento de raiz em algumas observações. A maior discrepância residual se deu nas estimativas até diâmetro do colo de $1,5 \mathrm{~mm}$ e a partir desse ponto há uma redução gradativa do percentual de erro apresentado pela equação. No ponto ideal de implantação, a maioria dos pontos mais concentrados próximo à linha zero.

A quantidade de raízes em uma muda é uma variável reconhecida como uma das melhores e mais importantes variáveis para a sobrevivência e estabelecimento das mudas em campo

FLORESTA, Curitiba, PR, v. 45, n. 4, p. 809 - 818, out. / dez. 2015.

Nicoletti, M. F. et al.

ISSN eletrônico 1982-4688 / ISSN impresso 0015-3826

DOI: $10.5380 /$ rf.v45i4.37454 
(BERNARDINO et al., 2005). Quanto maior a quantidade de raízes, maior será a superfície de absorção de nutrientes e consequentemente, melhor será o desenvolvimento das mudas (MAFIA et al., 2005). No entanto, como já citado, o uso desta variável na avaliação da qualidade de mudas em nível operacional é inviável, necessitando de modelos que se ajustem a essa variável, a partir de outras mais práticas e indestrutíveis, como altura e diâmetro do colo.

Ressaltando-se a necessidade de observar com maior rigor aspectos ambientais, como volume do tubetes, adubação, época do ano, densidade das mudas nas bandejas, idade da muda, entre outros. Esses fatores, além das próprias características da espécie, exercem grande influência nas variáveis morfológicas das mudas. Portanto, deve-se utilizar equações que se forneçam condições próprias para cada viveiro, já que as condições ambientais e de manejo variam em cada local de produção das mudas.

\section{Validação das equações}

Seguindo os critérios supramencionados, ressaltando a necessidade das mudas se enquadrarem na amplitude das variáveis morfológicas, além de semelhança dos fatores inerentes ao manejo das mudas no viveiro. $\mathrm{O}$ valor tabelado de Qui-quadrado corresponde ao valor limite de aceitação da hipótese de que os valores observados não diferem estatisticamente dos valores esperados, foi encontrado um $\chi^{2}$ tabelado igual a 28,9 .

Para a equação de área foliar, observou-se que as diferenças apresentadas entre os valores estimados e os valores mensurados geraram um valor de $\chi_{\text {calculado }}^{2}$ igual a 18,9. Pode-se, portanto, atestar validade das estimativas, uma vez que o valor calculado se apresenta dentro da região de aceitação (aceita-se a hipótese nula) para o nível de confiança de $99 \%$.

Comparando as estimativas da equação de comprimento de raiz com os valores mensurados,

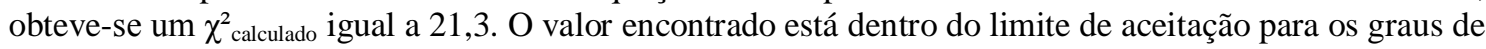
liberdade e nível de confiança propostos. Sendo assim, tornando a equação válida para estimativas de comprimento de raiz de mudas com características semelhantes às apresentadas no presente estudo.

\section{CONCLUSÕES}

- Com base nos resultados encontrados, pode-se afirmar que a área foliar e o comprimento radicular possuem alta correlação com as variáveis altura e diâmetro do colo.

- É possível utilizar equações para gerar estimativas de área foliar e comprimento de raiz em função de variáveis de fácil mensuração em mudas de Eucalyptus dunnii. Para a situação apresentada, propõese estimar a área foliar pela equação $a f=11,9334+2,5261 \cdot(h \cdot d c)+\varepsilon_{i}$ e para comprimento de raiz $c r=-2,0471-0,00028 \cdot h^{3}+4,3045 \cdot \ln (h) \cdot d c+\varepsilon_{i}$.

\section{REFERÊNCIAS}

AGUIAR, F. F. A.; KANASHIRO, S.; TAVARES, A. R.; NASCIMENTO, T. D. R.; ROCCO, F. M. Crescimento de mudas de pau-brasil (Caesalpinia echinata Lam.) submetidas a cinco níveis de sombreamento. Revista Ceres, Viçosa, v. 58, n. 6, p. 729 - 735, 2011.

ARAÚJO, D. D. Crescimento de mudas de Jatoba (Hymenaea courbaril) sob quatro níveis de sombreamento. 65 f. Dissertação (Mestrado em Botânica) - Universidade Federal Rural da Amazónia. Belém, PA. 2009.

BERNARDinO, D. C. S.; PAIVA, H. N.; NEVES, J. C. L.; GOMES, J. M.; MARQUES, V. B. Crescimento e qualidade de mudas de Anadenanthera macrocarpa (Benth.) em resposta à saturação por bases do substrato. Revista Árvore, Viçosa, v. 29, p. 863 - 870, 2005.

BONFIM, A. A. Qualidade de mudas de madeira-nova (Pterogyne nitens Tull) produzidas em tubetes e sacolas práticas e seu desempenho no campo. 70 f. Dissertação (Mestrado em Agronomia) Universidade Estadual do Sudoeste da Bahia. Vitória da Conquista, BA. 2007.

CARNEIRO, J. G. de A. Produção e controle de qualidade de mudas florestais. Curitiba, PR: UFPR/FUPEF. 451 p. 1995. 
D’AVILA, F. S.; PAIVA, H. N.; LEITE, H. G.; BARROS, N. F.; LEITE, F. P. Efeito do potássio na fase de rustificação de mudas clonais de eucalipto. Revista Árvore, Viçosa, v. 35, n. 1, p. 13 - 19, 2011.

DRAPER, N.; SMITH, H. Applied Regression Analysis. John Wiley, New York. 706 p. 1998.

DURVEA, M. L. Proceedings: Evaluating seedling quality: principles, procedures, and predictive abilities of major tests. 1985. Oregon State University, Corvallis.

ELOY, E.; CARON, B. O.; SCHMIDT, D.; BEHING, A.; SCHWERS, L.; ELLI, E. F. Avaliação da qualidade de mudas de Eucalyptus grandis utilizando parâmetros morfológicos. Floresta, v. 43, n. 3, p. 373 - 384, 2013.

LIMA, J. D.; SILVA, B. M. S.; MORAES, Q. S. Efeito da luz no crescimento de plântulas de Virola surinamensis (Rol.) Warb. Revista Científica Eletrônica de Engenharia Florestal, v. 10, n. 8, p. 1 - 10, 2006.

MAFIA, R. G.; ALFENAS, A. C.; SIQUEIRA, L.; FERREIRA, E. M.; LEITE, H. G.; CAVALLAZZI, J. R. P. Critério técnico para determinação da idade ótima de mudas de eucalipto para plantio. Revista Árvore, Viçosa, v. 29, p. 947 - 953, 2005.

PRODAN, M.; PETERS, R.; COX, F. Mensura forestal. San José, Costa Rica. Deutsche Gesellschaft für Techniche Zusammenarbeit (GTZ), Instituto Interamericano de Cooperación para la Agricultura (IICA), 586 p. 1997.

RUDEK, A.; GARCIA, F. A. O.; PERES, F. S. B. Avaliação da qualidade de mudas de eucalipto pela mensuração da área foliar com o uso de imagens digitais. Enciclopédia Biosfera, Centro Científico Conhecer, Goiânia, v. 9, n. 17, p. 3775. 2013.

WENDLING, I.; DUTRA, L. F. Produção de mudas de eucalipto por sementes. In: WENDLING, I.;DUTRA, L. F. Produção de mudas de eucalipto. Colombo: Embrapa Florestas, 2010. p. 13 - 47. 
FLORESTA, Curitiba, PR, v. 45, n. 4, p. 809 - 818, out. / dez. 2015.

Nicoletti, M. F. et al. et al. 